\title{
Arbitrary 3D Resolution Discrete Ray Tracing of Implicit Surfaces
}

\author{
Nilo Stolte \\ École de Technologie Supérieure, 1100 rue Notre-Dame Ouest, Montréal H30 1K3, Canada \\ nilo.stolte@online.fr
}

\begin{abstract}
A new approach to ray tracing implicit surfaces based on recursive space subdivision is presented in this paper. Interval arithmetic, already used to calculate intersections in ray tracing and ray casting (numerically or subdividing $1 \mathrm{D}$ or $2 \mathrm{D}$ spaces), is now used here to implement a ray tracing based on reliable rays traversals into a potentially infinite octree-like subdivided space, eliminating explicit intersections. Novel, robust and efficient algorithms for ray voxelization and BSP octant ordering are used to recursively traverse rays through the space. Implicit surfaces are robustly voxelized and hierarchically stored into an octree to a certain given level. During rendering, the subdivision based voxelization of surfaces and rays continues further down until a resolution near the discrete domain of the floating point numbers is acquired. To guarantee robustness of the ray voxelization, interval arithmetic with calculations performed under appropriate rounding modes in Pentium-4 x87 and SSE2 FPUs respectively is applied. The major advantage is that the traversal algorithm is guaranteed to find reliable intersections between the rays and the scene without any explicit intersection calculation, solving a known precision problem of the ray traversal in a previous approach, used here for comparison. The precision of the traversal can be arbitrarily increased within the limitation of the floating point representation.
\end{abstract}

\section{Introduction}

Ray tracing has been relying intensively on rays-objects intersections [1] which have been persistently imputed as the cause of its low efficiency. Acceleration techniques have been proposed to reduce in one way or another the number of intersection calculations. Some are based on space subdivision in which explicit ray-object intersections are bypassed by traversing rays through the subdivided space. Nonetheless, the ray traversal itself might rely on intersections between rays and bounding volumes [2, 3] or between rays and discrete subspaces where the scene was previously voxelized [4, 5, 6, 7]. Octrees $[8,4,6,5,7,9]$ have been proved to have great advantages for space decomposition in these techniques, since empty regions can be efficiently skipped. However, their advantages have been considerably hindered by the fact that the intersection calculations between rays and the boundaries of the regions traversed are not only inefficient but also unreliable [6]. Sometimes, these intersection calculations were implicitly or exclusively accomplished by incremental algorithms [8, 10,7,9]. Even though incremental algorithms are more efficient than direct intersection calculations they lack precision and are not reliable. As the discrete traversal advances, the mismatch between the continuous 
ray and the discrete counterpart considerably increases due to the lack of precision, thus parts of the scene would have the tendency to disappear at the end of the traversal since what the discrete ray intersects may be different from what the floating point ray intersects. Evidently, if the discrete traversal is done in low resolution spaces, as it is the case in most acceleration algorithms, the problem is less noticeable. However, the problem is particularly worse when considerably huge discrete spaces are traversed.

Prior to the algorithm presented in section 2 , robust ray traversals in discrete spaces have not yet been proposed to solve the problem, although there have been several robust techniques presented such as Lipshitz conditions or interval arithmetic. Interval arithmetic was introduced by Moore [11,12] and by Duff and Snyder [13, 14] into computer graphics. Since the problem of rounding errors can be very serious as seen in [15], the search for reliable algorithms for rendering is of quite significant importance. Although interval arithmetic has been used in several ray casting approaches [16, 14], the algorithm in section 2 is the first complete solution of an interval arithmetic based ray tracing with 3D space subdivision using discrete ray traversal. Kalra and Barr's ray tracing in [17] adopted a guaranteed ray intersection technique, that could not be considered a reliable solution for ray traversal. Their ray tracing [17] used Lipshitz conditions to voxelize implicit surfaces. However, explicit intersections between rays and octants as well as between rays and objects were still calculated. Duff's interval arithmetic ray casting [14] is robust, but it works in the image space and applies the perspective into the surface equations, thus being not compatible with space subdivision techniques.

In this article, a new approach is shown in which the traversal is done by voxelizing implicitly represented rays using the same technique to voxelize implicit surfaces. Surfaces and rays are simultaneously voxelized to avoid all explicit intersection calculations, between rays and objects as well as between rays and octants. The correlation between the discrete ray and the continuous ray is solved here because they are exactly the same. In this sense this approach resembles a discrete ray tracing [10, 9]. The basic differences in our approach are: (1) the voxelization of surfaces and rays are robust due to the use of interval arithmetic; (2) spatial resolution is much higher, allowing reaching the discrete domain of floating point numbers as proposed in [18]; (3) the scene is voxelized to a lower resolution into the octree as in [9] but during rendering the voxelization of surfaces and rays continues on the fly until a given precision is reached. Even though voxelization plays a crucial role, methods that do not ensure robustness [19, 20, 21] cannot be used in our context. By chance, implicit surfaces can be voxelized robustly [22, 14, 13, 23, 24] and a huge variety of forms and shapes can be defined implicitly.

Robust voxelization of implicit surfaces is generally implemented using spatial recursive subdivision [22, 14, 23, 24]. The methods are all conservative, though. Thus, spurious voxels might show up, depending on the surface, the voxelization method and how it is implemented. Although Lipshitz conditions can also be used to voxelize implicit surfaces [25], interval arithmetic is preferred in our approach because it is shown to be more efficient and more reliable [23]. To avoid the spurious regions, the implicit function describing the surface is evaluated at the eight vertices of the octants to verify if there is a change in sign when the last level is reached. Since it is only done at the 


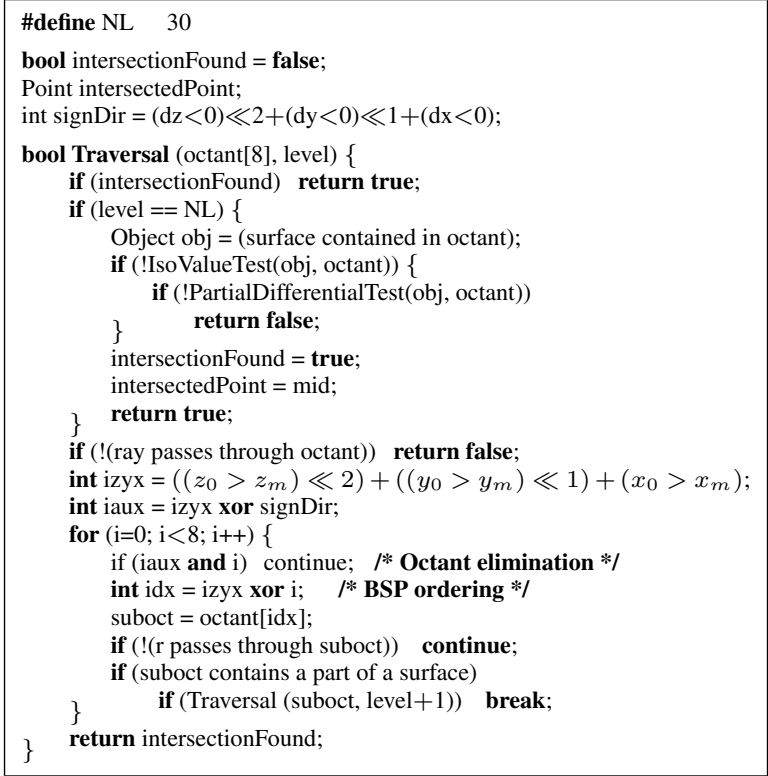

Fig. 1. Octree ray traversal algorithm

last level of the subdivision, the robustness throughout the process is guaranteed, but not in [17] because it is performed at each octant before testing the Lipschitz condition.

During the recursive subdivision at rendering time the order in which the octants are to be traversed is important. Our innovative BSP ordering algorithm ensures robustness in this process too. In this algorithm only the starting point of the ray and the middle point of the octant are required. The middle point is always an integer-like number that is produced by the addition of a power of two, half of the length of the octant plus the coordinate of the octant. This is guaranteed to avoid carry propagation, thus ensuring robustness as well as exactness. This calculation is always exact provided the half length of the octant is not smaller than $1 \mathrm{Ulp}$ (unit in the last place) [15].

\section{New Octree Ray Traversal Algorithm}

\subsection{Notations and Definitions}

Ray. The notation for the ray equation starting at $\left(x_{0}, y_{0}, z_{0}\right)$ and with $(d x, d y, d z)$ as its the direction vector is as follows:

$$
\left\{\begin{array}{l}
x=x_{0}+t \cdot d x \\
y=y_{0}+t \cdot d y \\
z=z_{0}+t \cdot d z
\end{array}\right.
$$

Scene. The scene is contained in an axis-aligned cube defined as the bounding box of all the surfaces. One of the cube's vertices is located at the origin $(0,0,0)$ and all the 
other ones have zero or positive coordinates. All the objects (surfaces) are defined within this cube, and previously voxelized using space subdivision.

Octant and Splitting Planes. The space subdivision starts splitting the scene into eight equal sized cubes, and each of these cubes is called an octant. An octant can be viewed as three intervals, each one along its respective coordinate axis:

$$
\left[x_{c}, X_{c}\right]\left[y_{c}, Y_{c}\right]\left[z_{c}, Z_{c}\right]
$$

where $\left(x_{c}, y_{c}, z_{c}\right)$, the vertex with lowest coordinates, is regarded as the coordinate of the octant, and $\left(X_{c}, Y_{c}, Z_{c}\right)$ is the vertex with highest coordinates. The subdivision of an octant is performed along the three axis-aligned splitting planes passing through the middle point of the octant, that is $\left(x_{m}, y_{m}, z_{m}\right)=\left(x_{c}+l / 2, y_{c}+l / 2, z_{c}+l / 2\right)$, where $l$ is the size of the octant. The splitting planes equations are then $x=x_{m}, y=y_{m}$ and $z=z_{m}$.

The eight subdivided octants (sub-octants) are stored in memory along an order determined by their relative locations in respect to the splitting planes, with indices from 0 to 7 . Actually each index consists of three bits, each corresponding to an axis. Each bit is set if the sub-octant lies on the positive side of the corresponding splitting plane, otherwise it is zero.

\subsection{Algorithm Overview}

The appealing idea of using discrete space resolutions so high to be able to reproduce the discrete domain of the floating point numbers to guarantee robustness [18] is for the first time implemented in this article. Moreover, the techniques in [24] to robustly voxelize different kinds of implicit surfaces are used as a basis in our approach to ray tracing. A conventional ray tracer is used here, while the intersection calculation is replaced by our new octree ray traversal algorithm.

During subdivision, each octant that might contain a part of a surface is further subdivided and so forth. Once a certain level is reached the octant is considered a leaf node or voxel and stored in an octree. During rendering time, the basic idea is to traverse each ray through the octree to find the first voxel containing a part of an object in the scene, which is in appearance similar to the work of Glassner [4] or Gargantini [6]. The fundamental difference between their approaches is that Glassner uses a linear octree, while Gargantini uses a hierarchical pointer octree. The approach in this article is different from their approaches in two aspects: (1) no explicit intersections between rays and octants neither between rays and objects are ever calculated; (2) the spatial recursive subdivision continues further down after the first voxel is found. This subdivision is done in the same way as the recursive voxelization, however, it is done on the fly and nothing needs to be stored, since rays and surfaces are voxelized concurrently. For each octant the eight sub-octants are sorted in the order that they might be traversed by the ray, and tested to verify if they are indeed traversed by the ray. This process is summarized in the pseudo code of Fig. 1. The IsoValueTest and PartialDifferentialTest appearing in Fig. 1 are discussed in section 2.5 .

In the implementations of Glassner's [4] and Gargantini's [6] approaches explicit intersection calculation is performed when traversing the subdivided space, which cannot 


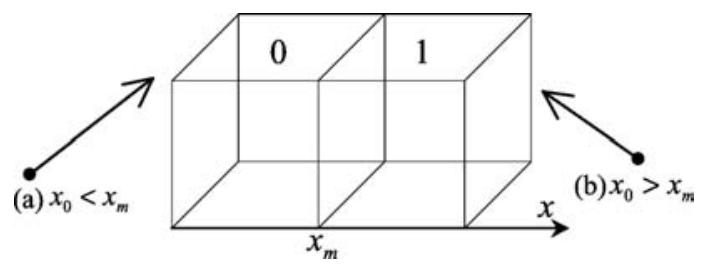

Fig. 2. Illustration of the BSP ordering technique

guarantee robustness. Our approach is significantly more reliable since the ray voxelization (see section 2.4) is robust and the BSP ordering scheme relies on calculations not affected by rounding errors. The traversal continues to recursively descend in the subdivided space whenever octants that satisfy the following three conditions are found:

1. The ray passes through the octant. This is tested using our new ray voxelization algorithm (see section 2.4).

2. The octant contains a part of a surface in the scene. When the traversal is within the octree, it is easily tested by verifying the data stored in the octree. When the traversal goes beyond the octree resolution, the implicit function inclusion function [13] used to voxelize the surface is reused to check this condition.

3. The octant is the nearest to $\left(x_{0}, y_{0}, z_{0}\right)$ satisfying conditions 1 and 2 . This is ensured using the BSP ordering technique (see section 2.3).

The traversal continues until an octant is finally found after descending $N L$ levels in the subdivided space, where $N L$ is the preset maximum traversal level, otherwise it is assumed that there is no intersection between the ray and the scene. The intersection point is then considered to be $\left(x_{m}, y_{m}, z_{m}\right)$. The precision obtained is dictated by the maximum distance between this point and the real intersection, that is, half of the octant diagonal length, which is $\sqrt{3} \cdot 2^{-N L-1}$. It can be seen that better precision can be obtained by increasing $N L$. For the IEEE 754 double precision numbers, the maximum value of $N L$ is 53, which is the number of bits in the significand including the hidden 1 , a binary digit always set to 1 except in special cases, which is not explicitly represented.

\subsection{Enhanced BSP Octant Ordering}

The BSP ordering technique is shown in Fig. 1 It is based on the relative location of the starting point of the ray in respect to the three splitting planes. This information is stored into the variable $i z y x$ as illustrated in Fig. 1 The variable $i$ stores the indexes of the octants as they appear in the memory, but these indexes are not in the front to back order as required in our ray traversal. As illustrated in Fig. 2, the memory voxel order is preserved in certain cases; otherwise it is reversed. Moreover, if the ray does not pass through a splitting plane, the four sub-octants at the opposite side of the splitting plane in respect to the starting point of the ray will never be traversed and are ignored. The efficiency of these two calculations comes from their extreme simplicity and reduced number of instructions. 


\subsection{Novel Robust Ray Voxelization Algorithm}

Ray Inclusion Function. Our method to test whether a ray passes through an octant is based on the implicit form of the ray. After eliminating the $t$ variable in (1) and applying a common factor $|d x| \cdot|d y| \cdot|d z|$, the implicit representation of the ray is obtained:

$$
c x \cdot\left(x-x_{0}\right)=c y \cdot\left(y-y_{0}\right)=c z \cdot\left(z-z_{0}\right)
$$

where

$$
\begin{aligned}
& c x=\operatorname{sign}(d x) \cdot|d y| \cdot|d z| \\
& c y=\operatorname{sign}(d y) \cdot|d z| \cdot|d x| \\
& c z=\operatorname{sign}(d z) \cdot|d x| \cdot|d y|
\end{aligned}
$$

Actually, each one of the three parts of (3) is the multiplication of $t$ in (1) by $|d x| \cdot|d y| \cdot|d z|$. The $\operatorname{sign}(d x), \operatorname{sign}(d y)$ and $\operatorname{sign}(d z)$ ensure that the values obtained from (3) have the same sign as the real $t$ in (1). Replacing the variables $x, y$ and $z$ in equation (3) by the three intervals in (2) produces one interval in each of the three parts of (3), as shown in (4).

$$
\begin{aligned}
& {\left[f_{x}\left(x_{c}\right), f_{x}\left(X_{c}\right)\right]=\left[c x \cdot\left(x_{c}-x_{0}\right), c x \cdot\left(X_{c}-x_{0}\right)\right]} \\
& {\left[f_{y}\left(y_{c}\right), f_{y}\left(Y_{c}\right)\right]=\left[c y \cdot\left(y_{c}-y_{0}\right), c y \cdot\left(Y_{c}-y_{0}\right)\right]} \\
& {\left[f_{z}\left(z_{c}\right), f_{z}\left(Z_{c}\right)\right]=\left[c z \cdot\left(z_{c}-z_{0}\right), c z \cdot\left(Z_{c}-z_{0}\right)\right]}
\end{aligned}
$$

As can be seen, the ray passes through the octant only if these three intervals overlap and this condition is what is used to test if a ray passes through an octant.

Using Correct Rounding Modes. The approach still suffers from rounding errors caused by the multiplications. The precision of the traversal is determined by the number of subdivision levels, each further subdivision corresponding to an additional bit of precision. In experiments where subdivision levels are close to the limits of floating point double precision (e.g. 50 levels), some octants are missed by rays that pass very close to octants' edges or vertices. In this case the two bounds of the octants in each axis differ only in the last few bits of the significand. Consequently, after applying them to (3) the resulting intervals are more prone to rounding errors. To guarantee robustness of this calculation, interval arithmetic with appropriate rounding modes is applied. The calculation in (4) is done with two rounding modes, towards ' $-\infty$ ' and ' $+\infty$ ', to the lower and upper bounds respectively. Consequently when the three intervals obtained with correct rounding modes do not overlap, it is assured that the ray does not pass through the octant. Moreover, we consider that there is no overlap when a lower bound of an interval is equal to the upper bound of another, since the ray in this case passes through neither one of them. To avoid penalties in performance normally involved in frequent change of rounding modes, each bound is calculated in a different floating point unit (FPU) preset with the required rounding mode. The Pentium-4 allows executing 2 double precision operations in SSE2 FPU and one double precision operation in the normal x87 FPU with independent rounding modes.

Optimizations. The actual implementation of this algorithm differs from what is described in Fig.1due to optimization reasons. The number of operations shown in section 


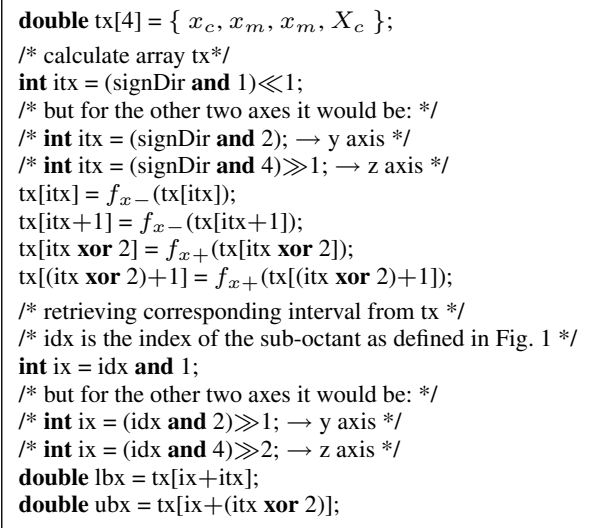

Fig. 3. Storing and retrieving the interval bounds with correct rounding modes without testing to avoid stalling the processor pipeline

2.6 takes these optimizations into account. In Fig. 1, the test to verify if a ray passes through an octant is carried out once for the octant itself and once for each of its suboctants, thus giving rise to 9 tests involving the evaluation of 27 intervals. Since they share bounds with each other, only 3 intervals along each axis are really considered for testing all the 9 octants, so only 9 of the 27 intervals are indeed calculated. To avoid these repetitions all the eight sub-octants and their parent octant are tested together. What remains to be elucidated is the selection of the correct rounding mode for each bound of an interval. For the $x$ coordinate, the following three intervals, $\left[x_{c}, X_{c}\right],\left[x_{c}, x_{m}\right]$ and $\left[x_{m}, X_{c}\right]$ will be applied to the implicit ray equation (3), and three resulting intervals will be obtained. However, the proper rounding modes to guarantee numerical robustness depend on the direction of the ray along the $\mathrm{x}$ axis $(d x)$. In the case that $d x>0$, the resulting intervals are

$$
\left[f_{x-}\left(x_{c}\right), f_{x+}\left(X_{c}\right)\right],\left[f_{x-}\left(x_{c}\right), f_{x+}\left(x_{m}\right)\right],\left[f_{x-}\left(x_{m}\right), f_{x+}\left(X_{c}\right)\right]
$$

where $f_{x-}()$ and $f_{x+}()$ represent the calculation of $f_{x}()$ in (4) using rounding modes towards ' $-\infty$ ' and ' $+\infty$ ' respectively. When $d x<0$, the resulting intervals will be

$$
\left[f_{x-}\left(X_{c}\right), f_{x+}\left(x_{c}\right)\right],\left[f_{x-}\left(X_{c}\right), f_{x+}\left(x_{m}\right)\right],\left[f_{x-}\left(x_{m}\right), f_{x+}\left(x_{c}\right)\right]
$$

Evidently $x_{c}$ and $X_{c}$ are calculated only once under different rounding modes, and $x_{m}$ is always calculated twice, each one under a different rounding mode respectively. Therefore, an array of four elements $t x[4]$ is used to represent the three resulting intervals. When $d x>0, t x[0]$ and $t x[1]$ are calculated with rounding mode 'towards $-\infty$ ', and $t x[2]$ and $t x[3]$ are calculated with rounding mode 'towards $+\infty$ '; or vice versa when $d x<0$. The same logic is used along $\mathrm{y}$ and $\mathrm{z}$ axis. To avoid tests and branch instructions that may stall the processor pipeline, the indices of the elements in the arrays to store the values calculated under each rounding mode are automatically selected. A similar scheme is used to retrieve the correct lower and upper bounds stored in $t x$ array for each sub-octant. The procedure is shown in Fig. 3 . 


\subsection{Partial Differential Test}

The robustness of interval arithmetic can guarantee that no parts of the surfaces were omitted during voxelization/traversal. On the other hand, however, it cannot guarantee that each leaf voxel really contains a part of a surface, thus resulting in overestimations during the voxelization/traversal process. To evaluate and reduce these overestimations, two algorithms as proposed in [18] are applied on the final octant and the surface contained in it. The first one calculates the eight iso-values of the function corresponding to the surface at the eight corners of the octant, and checks if there is a change in sign. This algorithm may suffer from rounding errors as suggested in [18], since it directly uses the surface equation. In some cases, these rounding errors will possibly be added to the half of the octant diagonal length error described at the end of section 2.2 Even though it is possible to solve the problem, the reliability at the leaf level is already quite high; therefore, the use of this algorithm is acceptable.

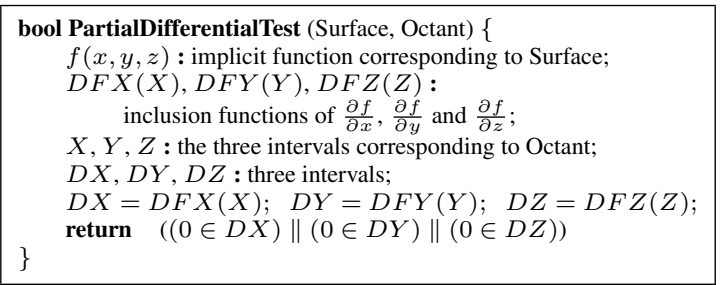

Fig. 4. Partial differential test of an octant

If there is a change in sign, the octant is considered to be correct at the given precision, otherwise the second algorithm will be applied, which examines the monotonicity of the implicit function as shown in Fig. 44 If the partial differential test returns true, the function is monotonic within the $x, y$, and $z$ range of the octant, thus the function has no zeroes within the octant, so the octant is ignored and the traversal continues. If the test returns false, the traversal stops, despite that in very special cases, this octant may not contain a part of a surface. This case does not occur in any surface ray traced in this paper; however, it needs to be considered in future work.

\subsection{Comparison with Gargantini's Algorithm}

Octree traversal algorithms, such as Gargantini's [6], calculate intersections between the ray and the octants it traverses. Therefore, the traversal algorithm in section 2.4 is compared with Gargantini's. The rest of the program is exactly the same.

Gargantini's algorithm exploits the fact that a ray can pass through at most four sub-octants in an octant. Assuming that the entry and exit points of a ray into an octant are known, intersections between the ray and all three subdivision mid-planes are then calculated using (1). Only the $m(m \leq 3)$ intersected points that are within the octant are retained and sorted in ascending order according to their corresponding $t$ values, resulting in $m+1$ ray segments. The lower and upper bounds of each segment correspond to the 
entry and exit points of the ray into one sub-octant, thus the sub-octants traversed by the ray are obtained in the correct order. The technique above was described in [6]. Due to differences between the octants' structures, the implementation here has some variations in respect to [6]. Here, an octant only contains an array of pointers to its sub-octants. After the index of a sub-octant is known, its coordinates and then its middle point need to be calculated. In [6] only the indices of the sub-octants are needed, since their coordinates are not used, and the octant structure already contains the middle point coordinates. Because of the variations in design, the number of operations of Gargantini's algorithm contains more floating point additions and bit operations than what was claimed in [6]. The extra data cannot be stored in our case since our traversal is also done on the fly as well as in the octree, while in [6] it is limited to the octree.

The advantage of the traversal algorithm in [6] is that it eliminates all the suboctants that are not traversed by the ray. Comparatively, the BSP ordering and auxiliary techniques in our algorithm can only partially eliminate them, whereas the remaining ones are still tested by the ray voxelization algorithm in section 2.4 However, as can be seen from the results in section 3 , the traversal algorithm in this article is a bit faster than Gargantini's, and is just slightly slower after performing the calculations using robust interval arithmetic with different rounding modes. This is due to the high efficiency of our BSP ordering and ray voxelization techniques.

If the precision/resolution is not too high (e.g. 30 levels), the traversals in both Gargantini's and our approach are exactly the same for the scenes tested. However, when subdivision levels become close to the limits of the floating point precision the two traversals start to mismatch. To verify which traversal is correct, a 128 bits precision binary floating point arithmetic package was used. A verification program using this package calculates the intersections between a ray and each sub-octant of a traversed octant, giving the sub-octants indices in the order they are traversed. In all the scenes tested our approach applying interval arithmetic with correct rounding modes (with SSE2) always exhibited traversals identical to the ones obtained by the verification program. Gargantini pointed out in [6] the two cases when the errors may occur, and described a method to avoid the choice of incorrect octant under certain conditions restricted to ray casting. However, the method is not a complete solution for all cases, and can only eliminate a part of the errors. Comparatively, after applying interval arithmetic with correct rounding modes, the algorithm described in section 2.4 can guarantee that an octant will never be missed without much effect in the performance, thus solving the problem in Gargantini's algorithm.

\section{Results}

Table 1 shows the times for generating $512 \times 512$ images. Six kinds of surfaces are ray traced using a PC with a Pentium-4 $2.4 \mathrm{GHz}$ processor and 512Mbytes of main memory. Their equations can be seen in Table 1. For each kind of surface, images were generated for both ray casting and ray tracing, using Gargantini's algorithm, our algorithm with and without proper rounding modes using SSE2 instructions respectively, see Fig. 5 

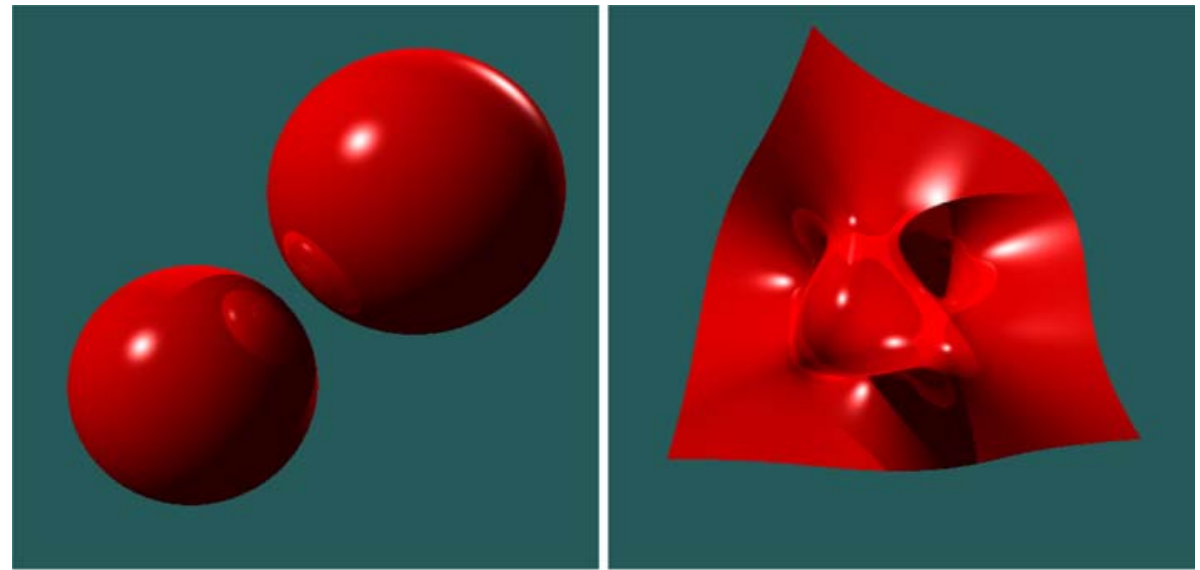

(a) Spheres

(b) Holesrot

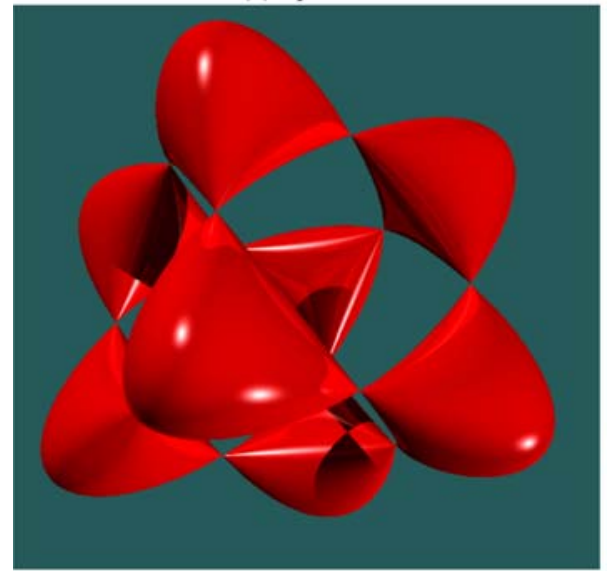

(c) Quartdef
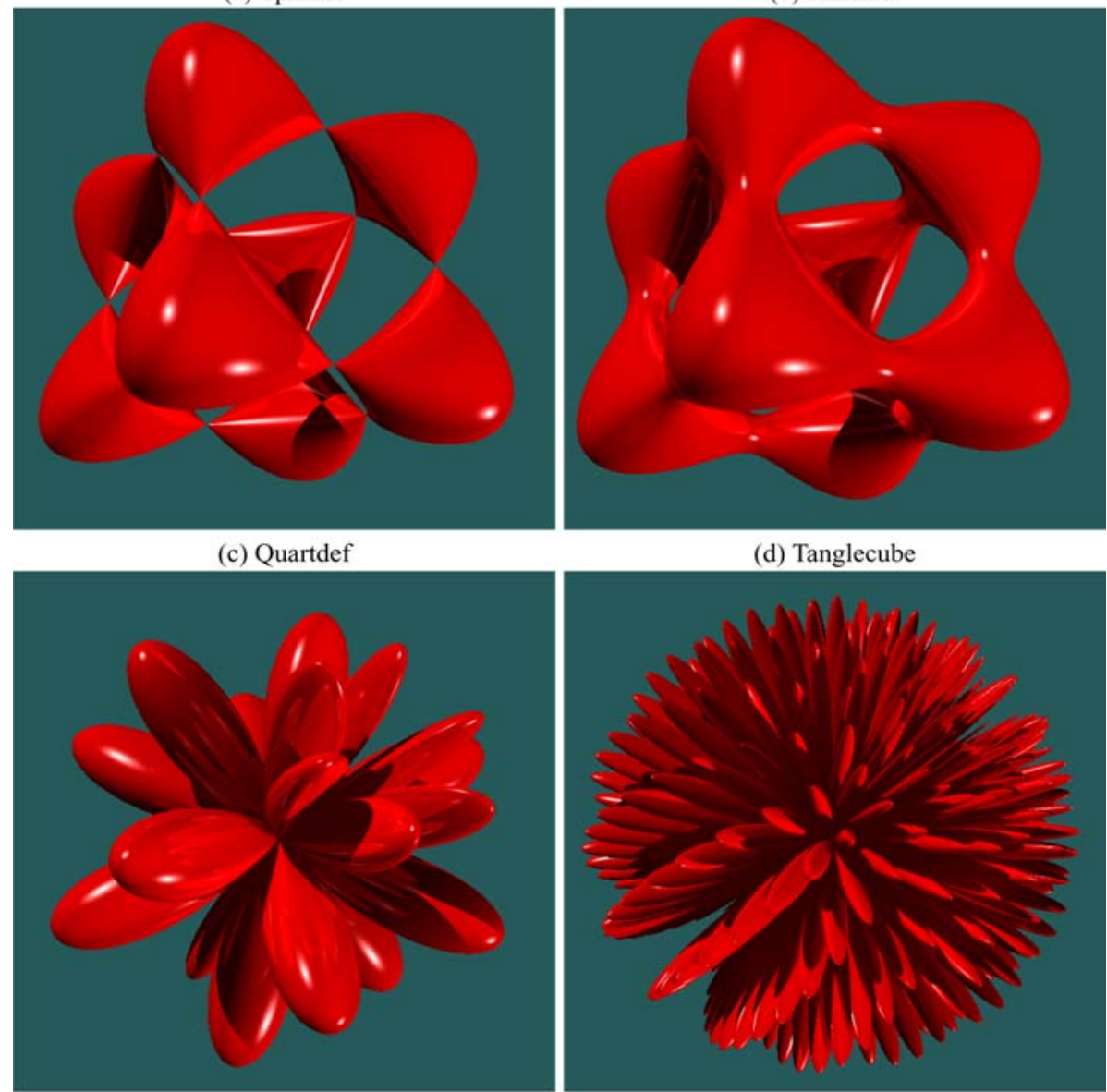

(d) Tanglecube

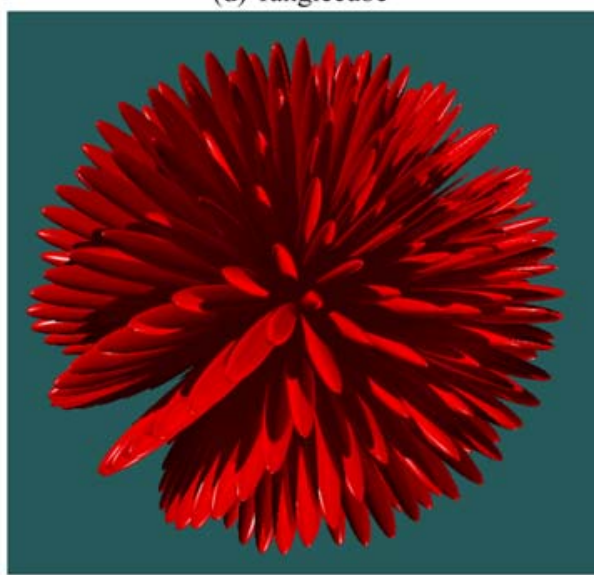

(e) Asterisk $(\mathrm{m}=3, \mathrm{n}=4)$

(f) Asterisk(m=9, n=18)

Fig. 5. Ray tracing results with 5 level reflections 
Table 1. Ray tracing times $(\mathrm{sec})$ for $512 \times 512$ images, 30 bits precision, 2 light sources and 5 levels of reflections (except for ray casting)

\begin{tabular}{|l|l|r|r|r|}
\hline \hline Primitive & Equations & Gar. & \multicolumn{1}{c|}{ Ours } & with SSE2 \\
\hline \hline spheres \\
ray casting & & 21.672 & 20.687 & 27.062 \\
\hline quartdef \\
ray casting & $\left(x^{2}-1\right)^{2}+\left(y^{2}-1\right)^{2}+\left(z^{2}-1\right)^{2}-1=0$ & 15.516 & 15.235 & 19.860 \\
\hline tanglecube & & 37.609 & 35.140 & 45.281 \\
ray casting & $x^{4}-5 x^{2}+y^{4}-5 y^{2}+z^{4}-5 z^{2}+11.8=0$ & 46.737 & 43.015 & 31.891 \\
\hline holesrot & & 43.719 & 28.813 & 37.531 \\
ray casting & $x^{3}+y^{3}+z^{3}-x-y-z=0$ & 30.469 & 29.313 & 53.500 \\
\hline asterisk(3,4) & & 204.327 & 193.233 & 209.608 \\
ray casting & $\sin (3 \theta) \sin (4 \phi)-R=0$ & 113.875 & 109.546 & 118.750 \\
\hline $\begin{array}{l}\text { asterisk(9,18) } \\
\text { ray casting }\end{array}$ & $\sin (9 \theta) \sin (18 \phi)-R=0$ & 435.638 & 410.841 & 445.982 \\
\hline
\end{tabular}

The surfaces are voxelized at octree level 9 ( $512^{3}$ resolution), with $N L=30$. Only images generated by our algorithm with SSE2 are shown since the images have no visible differences in comparison to those generated by Gargantini's.

It can be seen from Table 1 that our algorithms exhibit similar performance as Gargantini's. The one using interval arithmetic under proper rounding modes (with SSE2 instructions) is slightly slower, whereas the one without rounding (Ours) is several seconds faster (see discussion in section 2.6). Gargantini's algorithm was compared with Samet's in [6], and the results showed that the time in Gargantini's approach is nearly half of the time of Samet's. Thus our approach is also fairly twice faster than Samet's.

Analyzing the performance based on the number of rays per second (rays/sec), one concludes that the surfaces with tight inclusion functions exhibit roughly the same performance, thus suggesting the algorithm is insensitive to the surfaces' complexity. The best performance was with spheres, since they have quite simple equations and also the tightest inclusion functions. Naively applying interval arithmetic in the tanglecube and the holesrot did not originally provide tight inclusion functions. Cleverly decomposing their expressions led to quite tight inclusion functions significantly enhancing the performance. With the optimizations (shown in the times in the Table 1), the performance of the holesrot almost doubles, whereas the tanglecube is almost 4 times faster.

\section{Conclusion}

A new algorithm is here shown to ray trace implicit surfaces without explicit intersection. The intersection estimation converges in $O\left(\log _{8} N\right)$, where $N$ is the number of voxels of the discrete space $\left(2^{3 N L}\right)$. It works by voxelizing rays and objects by recursively subdividing the space and using interval arithmetic to discard regions not crossed by a ray or a surface. A novel BSP octant ordering technique is used to efficiently traverse the rays; it is robust since the values involved are exact. Both the ray and object voxelizations are also robust, thus guaranteeing the reliability. A partial differential test algorithm is 
sometimes applied to eliminate overestimations of interval arithmetic. The results show that the algorithm is insensitive to the surfaces' complexity but quite sensitive to the inclusion functions tightness, since different surfaces with similar inclusion function tightness exhibit similar rays/sec performance.

\section{References}

1. Whitted, T.: An Improved Ilumination Model for Shaded Display. Communications of the ACM 23 (1980) 343-349

2. Rubin, S.M., Whitted, T.: A 3-Dimensional Representation for Fast Rendering Complex Scenes. Computer Graphics 14 (1980) 110-116

3. Kay, T., Kajiya, J.: Ray Tracing Complex Scenes. Computer Graphics 20 (1986) 269-278

4. Glassner, A.S.: Space Subdivision for Fast Ray Tracing. IEEE - CGA 10 (1984) 15-22

5. Jevans, D., Wyvill, B.: Adaptative Voxel Subdivision for Ray Tracing. In: Proceedings of Graphics Interface '89, Toronto, Ontario, Canadian Information Processing Society (1989) 164-172

6. Gargantini, I.: Ray tracing an Octree: Numerical Evaluation of the First Intersection. Computer Graphics forum 12 (1993) 199-210

7. Endl, R., Sommer, M.: Classification of Ray-Generators in Uniform Subdivisions and Octrees for Ray Tracing. Computer Graphics forum 13 (1994) 3-19

8. Fujimoto, A., Tanaka, T., Iwata, K.: ARTS: Accelerated Ray Tracing System. IEEE - CGA 6 (1986) 16-26

9. Stolte, N., Caubet, R.: Discrete Ray-Tracing of Huge Voxel Spaces. Computer Graphics Forum 14 (1995) 383-394

10. Yagel, R., Cohen, D., Kaufman, A.: Discrete Ray Tracing. IEEE - CGA 12 (1992) 19-28

11. Moore, R.E.: Interval Analysis. Prentice-Hall, Englewood Cliffs, NJ (1966)

12. Moore, R.E.: Methods and Application of Interval Analysis. Society for Industrial and Applied Mathematics (SIAM), Philadelphia (1979)

13. Snyder, J.M.: Interval Analysis For Computer Graphics. Computer Graphics 26 (1992) $121-130$

14. Duff, T.: Interval Arithmetic and Recursive Subdivision for Implicit Functions and Constructive Solid Geometry. Computer Graphics 26 (1992) 131-138

15. Goldberg, D.: What Every Computer Scientist Should Know About Floating-Point Arithmetic. ACM Computing Surveys 23 (1991) 5-48

16. de Cusatis Junior, A., de Figueiredo, L.H., Gattass, M.: Interval Methods for Ray Casting Implicit Surfaces with Affine Arithmetic. SIBGRAPHI (1999) 17-20

17. Kalra, D., Barr, A.: Guaranteed Ray Intersections with Implicit Surfaces. Computer Graphics 23 (1989) 297-306

18. Stolte, N.: High Resolution Discrete Spaces: A New Approach for Modeling and Realistic Rendering (Espaces Discrets de Haute Résolutions: Une Nouvelle Approche pour la Modelisation et le Rendu d'Images Réalistes). PhD thesis, Université Paul Sabatier - Toulouse France (1996)

19. Kaufman, A.: An Algorithm for 3D Scan-Conversion of Polygons. In: Eurographics'87, Amsterdam, North Holand (1987) 197-208

20. Kaufman, A.: Efficient Algorithms for 3D Scan-Conversion of Parametric Curves, Surfaces, and Volumes. Computer Graphics 21 (1987) 171-179

21. Greene, N.: Voxel Space Automata: Modeling with Stochastic Growth Processes in Voxel Space. Computer Graphics 23 (1989) 175-184

22. Taubin, G.: Rasterizing Algebraic Curves and Surfaces. IEEE - CGA (1994) 14-23 
23. Stolte, N., Caubet, R.: Comparison between different Rasterization Methods for Implicit Surfaces. In Rae Earnshaw, John A. Vince and How Jones, ed.: Visualization and Modeling. Academic Press (1997) 191-201

24. Stolte, N., Kaufman, A.: Novel Techniques for Robust Voxelization and Visualization of Implicit Surfaces. Graphical Models 63 (2001) 387-412

25. Bidasaria, H.B.: Defining and Rendering of Textured Objects through The Use of Exponential Functions. Graphical Models and Image Processing 54 (1992) 97-102 